\title{
Outcome of relapsed or refractory acute B-lymphoblastic leukemia patients and $B C R-A B L$-positive blast cell crisis of B-lymphoid lineage with extramedullary disease receiving inotuzumab ozogamicin
}

Sabine Kayser, ${ }^{1,2}$ Chiara Sartor, ${ }^{3}$ Marlise R. Luskin, ${ }^{4}$ Jonathan Webster, ${ }^{5}$ Fabio Giglio, ${ }^{6}$ Nydia Panitz, ${ }^{1}$ Andrew M. Brunner, ${ }^{7}$ Matthias Fante, ${ }^{8}$ Christoph Lutz, ${ }^{9,10}$ Daniel Wolff, ${ }^{8}$ Anthony D. Ho, ${ }^{9}$ Mark J. Levis, ${ }^{5}$ Richard F. Schlenk ${ }^{2,9 \#}$ and Cristina Papayannidis ${ }^{11 \#}$

${ }^{1}$ Medical Clinic and Policlinic I, Hematology and Cellular Therapy, University Hospital Leipzig, Leipzig, Germany; ${ }^{2}$ NCT Trial Center, National Center of Tumor Diseases, German Cancer Research Center (DKFZ), Heidelberg, Germany; ${ }^{3}$ stituto di Ematologia "Seràgnoli",

Dipartimento di Medicina Specialistica, Diagnostica e Sperimentale, Università degli Studi, Bologna, Italy; ${ }^{4}$ Department of Medical Oncology, Dana-Farber Cancer Institute, Boston, MA, USA; ${ }^{5}$ Sidney Kimmel Comprehensive Cancer Center, Johns Hopkins University, Baltimore, MD, USA; ${ }^{6} \mathrm{Hematology}$ and Bone Marrow Transplantation Unit, San Raffaele Scientific Institute, Milan, Italy; ${ }^{7}$ Massachusetts General Hospital, Boston, MA, USA; ${ }^{8}$ Department of Hematology and Oncology, Internal Medicine III, University Hospital Regensburg,

Regensburg, Germany; ' $D$ Department of Internal Medicine V, Heidelberg University Hospital, Heidelberg, Germany; ${ }^{10}$ Praxis for Hematology and Oncology Koblenz, Koblenz, Germany and ${ }^{11}$ IRCCS Azienda Ospedaliero-Universitaria di Bologna, Istituto di Ematologia "Seràgnoli" Bologna, Italy.
Correspondence: S. Kayser s.kayser@dkfz-heidelberg.de

Received: November 29, 2021. Accepted: $\quad$ February 1, 2022.

Prepublished: February 10, 2022

https://doi.org/10.3324/haematol.2021.280433

๑2022 Ferrata Storti Foundation Published under a CC BY-NC license (c) (1) $($ )

${ }^{\#} R F S$ and CP contributed equally as co-senior authors.

\begin{abstract}
Acute lymphoblastic leukemia (ALL) can relapse in the extramedullary compartment, with or without medullary involvement. Response to treatment may be individual. We evaluated response to inotuzumab ozogamicin in 31 patients with relapsed/refractory B-ALL with extramedullary disease. Median age was 31 years (range, 19-81). All patients were heavily pretreated, including allogeneic hematopoietic stem cell transplantation (HSCT; $n=18$ ). Overall response rate after two cycles of inotuzumab ozogamicin was 84\% (complete remission, 55\%; partial remission, 29\%; resistant disease, 13\%; early death, 3\%). The median follow-up was 29 months and median overall survival was 12.8 months. One-year and 2-year overall survival rates were $53 \%$ (95\% Cl: $37-76 \%)$ and $18 \%$ (95\% Cl: $8-43 \%)$, respectively. Age had no impact on overall survival when assessed as a continuous variable or dichotomized at 60 years. Twelve patients proceeded to allogeneic HSCT (complete remission, $n=6$; partial remission, $n=3$; resistant disease, $n=3$ ). Prior to allogeneic HSCT, eight patients received two or fewer cycles and four patients received three or four cycles of inotuzumab ozogamicin. Sinusoidal obstruction syndrome was reported in three patients, including one after transplantation. Allogeneic HSCT, evaluated as a time-dependent variable, had no impact on overall survival. Inotuzumab ozogamicin seems to be effective as a debulking strategy in relapsed/refractory ALL with extramedullary disease. However, inotuzumab ozogamicin followed by allogeneic HSCT seems not to be effective in maintaining long-term disease control.
\end{abstract}

\section{Introduction}

Historically, refractory/relapsed ( $r / r)$ B-cell acute lymphoblastic leukemia (B-ALL) in adults has a dismal prognosis, with less than $10 \%$ of patients being long-term survivors. ${ }^{1}$ At present, allogeneic hematopoietic stem cell transplantation (HSCT) is considered the only curative option for patients with $r / r$ B-ALL with best outcomes achieved after effective salvage re-induction therapy and transplantation in complete remission (CR) without measurable residual disease. ${ }^{2,3}$

The role of novel immune-based chimeric antigen receptor T-cell infusions in this setting has remained undefined. ${ }^{4-6}$ Although conventional salvage chemotherapy 
is capable of inducing CR rates of $18 \%$ to $44 \%$ in patients with $\mathrm{r} / \mathrm{r}$ B-ALL, ${ }^{7-13}$ antibody-based strategies using blinatumomab or inotuzumab ozogamicin (INO) have been proven to be more effective. ${ }^{14,15}$ INO is a humanized anti-CD22 monoclonal antibody conjugated to the potent cytotoxic agent calicheamicin, which was developed as a targeted therapy for B-cell malignancies. ${ }^{16,17}$ Upon binding to CD22 and internalization, calicheamicin is off-set and binds to DNA, thereby leading to double-strand breaks and apoptosis. ${ }^{16,17}$

The phase III INO-VATE trial demonstrated that INO had superior efficacy compared to standard-of-care treatment for $\mathrm{r} / \mathrm{r} \mathrm{B}-\mathrm{ALL}$, inducing CR/CR with incomplete hematologic recovery (CRi) in $80.7 \%$ and $29.4 \%$ of the patients, respectively $(P<0.001){ }^{15}$ Additionally, the rate of negativity for measurable residual disease $(0.01 \%$ marrow blasts assessed at a central laboratory by multicolor, multiparameter flow cytometry) in patients with $\mathrm{CR} / \mathrm{CRi}$ was significantly higher after treatment with INO than after standard-of-care $(78.4 \%$ vs. $28.1 \%$; $P<0.001)$. After INO treatment, $41 \%$ of patients proceeded directly to allogeneic HSCT as compared to $11 \%$ after standard-of-care $(P<0.001)$. The median progression-free survival was significantly longer after INO than after standard-ofcare (5.0 months vs. 1.8 months; $P<0.001)$. The median overall survival (OS) was 7.7 months after INO as compared to 6.2 months after standard-of-care, and the 2-year OS rates were $23 \%$ versus $10 \%$, respectively. ${ }^{15}$ The most frequent grade 3 or higher non-hematologic adverse events after INO were liver-related. Veno-occlusive liver disease (VOD)/sinusoidal obstruction syndrome (SOS) of any grade occurred in 15 patients (11\%), who received INO and in one patient (1\%) after standard-of-care therapy. In addition, ten of 48 (21\%) patients, who underwent allogeneic HSCT after INO treatment, developed VOD after transplantation; three of these ten patients had received a second transplant. ${ }^{15}$ Deep remissions with negativity for measurable residual disease can be achieved with INO treatment in patients with $\mathrm{r} / \mathrm{r}$ ALL. However, the safety and efficacy of INO treatment in patients with $r / r$ ALL and extramedullary disease (EMD) is currently unclear. Patients with central nervous system infiltration and/or isolated EMD were excluded from the phase III randomized INO-VATE trial. ${ }^{15}$ Of note, extramedullary relapses are common in $\mathrm{r} / \mathrm{r}$ ALL patients following exposure to blinatumomab, occurring in up to $40 \% .18,19$

EMD in $\mathrm{r} / \mathrm{r} \mathrm{B}-\mathrm{ALL}$ is characterized by a dismal outcome with no accepted standard therapeutic approaches.' The objectives of our study were to characterize a series of adult $r / r$ B-ALL patients with EMD and evaluate their outcome after treatment with INO.

\section{Methods}

\section{Patients}

Information on 31 adult patients (median age, 31 years; range,
19-81 years) with histologically confirmed $\mathrm{r} / \mathrm{r} \mathrm{B}-\mathrm{ALL}$ and EMD, who were treated with INO between 2015 and 2021 within a compassionate use program $(n=7)$ or in-label after approval by the Food \& Drug Administration or the European Medical Agency $(n=24)$ was collected from six institutions in the USA and Europe. All 31 patients were CD22-positive at relapse/progressive disease. Three (10\%) of the 31 patients had been previously treated with tyrosine kinase inhibitors for chronic myeloid leukemia and progressed to BCR-ABLpositive blast cell crisis of $B$-lymphoid lineage. Bone marrow evaluation and immunophenotyping by flow cytometry revealed B-ALL in all three patients. The 31 patients were heavily pretreated having received intensive chemotherapy with or without a tyrosine kinase inhibitor, as well as blinatumomab in 14, and local irradiation in five patients. In addition, allogeneic HSCT had been performed in 18 patients (first-line or at relapse, $n=9$, each).

Participating centers were chosen upon network relationships of the first and last authors. Detailed case report forms (including information on baseline characteristics, chemotherapy, allogeneic HSCT, response, and survival) were collected from all participating centers. Inclusion criteria were adult patients with $r / r$ ALL and EMD. All patients who fulfilled these criteria were included by the participating institutions.

Chromosome banding was performed using standard techniques, and karyotypes were described according to the International System for Human Cytogenetic Nomenclature..$^{20}$ Data collection and analyses were approved by the Institutional Review Boards of the participating centers.

\section{Treatment}

INO was administered at a dose of $0.8 \mathrm{mg} / \mathrm{m}^{2}$ body surface area as a continuous intravenous infusion over $1 \mathrm{~h}$ on day 1 and at $0.5 \mathrm{mg} / \mathrm{m}^{2}$ body surface area on days 8 and 15. Once the patients had achieved CR, the dose on day 1 of each consecutive cycle was reduced to $0.5 \mathrm{mg} / \mathrm{m}^{2}$ body surface area. Up to six INO cycles ( $\leq 2$ cycles, $n=19 ; 3-4$ cycles, $n=7 ; 5-6$ cycles, $n=5$ ) were administered according to the previously approved regimen. The three patients with $B C R$-ABL-positive blast cell crisis of $\mathrm{B}$-lymphoid lineage received a tyrosine kinase inhibitor in addition to INO. EMD response was assessed by computed tomography (CT) or positron emission tomography-computed tomography (PET-CT). VOD/SOS was assessed according to previously defined clinical criteria and diagnosed by the treating investigator. ${ }^{15}$

\section{Statistical analyses}

Patients' characteristics were compared with the KruskalWallis rank sum test for continuous variables and the Fisher exact test for categorical variables. The median follow-up time was computed using the reverse Kaplan-Meier estimate..$^{21}$ The Kaplan-Meier method was used to estimate the 
distribution of relapse-free survival and OS. ${ }^{22}$ OS was calculated from the start of INO treatment until last followup or death. Relapse-free survival was calculated from achievement of CR after the start of INO treatment until last follow-up or relapse. The confidence interval (Cl) estimation for survival curves was based on the cumulative hazard function using the Greenwood formula for variance estimation. Log-rank tests were employed to compare survival curves between groups. The effect of allogeneic HSCT on OS as a time-dependent intervening event was tested using the Mantel-Byar method ${ }^{23}$ for univariable and Andersen-Gill model for multivariable analyses. ${ }^{24}$ The method of Simon and Makuch was used to estimate survival distributions with respect to time-dependent interventions. ${ }^{25}$ The individuals at risk were initially all represented in the INO therapy group. If patients underwent allogeneic HSCT, they were removed at this time point from the INO therapy group and further followed up within the allogeneic HSCT group. All statistical analyses were performed with the statistical software environment $R$, version 3.3.1, using the $R$ packages prodlim, version 1.5.7, and survival, version $2.39-5 .{ }^{26}$

\section{Results}

\section{Patients' characteristics}

At the time of $r / r$ ALL with EMD, median white blood cell and platelet counts were $5.9 \times 10^{9} / \mathrm{L}$ (range, $0.04-36 \times 10 \% / \mathrm{L}$ ) and $110.5 \times 10^{9} / \mathrm{L}$ (range, $6-337 \times 10^{\%} / \mathrm{L}$ ), respectively. Fifteen patients (48\%) were female; Eastern Cooperative Oncology Group score was $\leq 2$ in 29 patients and 3 in two patients (Table 1). Overall, patients had a median of two EMD manifestations (range, 1-9). Localization of EMD is shown in Table

Table 1. Patients' characteristics at the time-point of relapsed/refractory acute lymphoblastic leukemia and extramedullary disease.

\begin{tabular}{|l|c|c|}
\hline & Number (31) & $\%$ \\
\hline Female gender & 15 & 48 \\
\hline $\begin{array}{l}\text { ECOG status } \\
\leq 2\end{array}$ & 29 & \\
3 & 2 & 94 \\
\hline $\begin{array}{l}\text { Median age, years } \\
\text { Median WBC x10\%/L } \\
\text { missing }\end{array}$ & Value & Range \\
\hline $\begin{array}{l}\text { Platelets } \times 10 \% / L \\
\text { missing }\end{array}$ & 31 & $19-81$ \\
\hline $\begin{array}{l}\text { Hemoglobin, g/dL } \\
\text { missing }\end{array}$ & 5.9 & $0.04-36$ \\
\hline $\begin{array}{l}\text { Median BM blast cells, \% } \\
\text { missing }\end{array}$ & 110.5 & $6-337$ \\
\hline
\end{tabular}

ECOG: Eastern Cooperative Oncology Group; WBC: white blood cell count; BM: bone marrow.
2. In addition to EMD, 16 (52\%) patients had a relapse in bone marrow.

\section{Genetics}

Cytogenetic analysis at the time of $r / r$ ALL with EMD was available for 13 (42\%) patients, of whom six had a bone marrow relapse as well. Of the 13 patients with cytogenetic information, six had a normal karyotype, four had a complex karyotype ( $\geq 3$ abnormalities), two had a $\mathrm{t}(9 ; 22)(q 34 ; q 11)$ and one had an additional X-chromosome. In one patient clonal evolution to a complex karyotype was detected.

\section{Response}

Response was not assessed after the first induction cycle in seven (23\%) of the 31 patients, including one who died at day 11 of the first INO cycle due to cerebral hemorrhage. Figures 1 and 2 are representative PET-CT images of patients with partial remission (PR) and $C R$, respectively. CR assessed by PET-CT (CR; including EMD and hematologic/bone marrow CR) after the first INO cycle was achieved in ten of the 24 assessed patients (42\%) (Figure $2 \mathrm{~A}, \mathrm{~B}$ ), nine patients (37.5\%) had a PR (Figure $1 \mathrm{~A}, \mathrm{~B}$ ), two $(8 \%)$ had stable disease and three $(12.5 \%)$ showed resistant/progressive disease. After two cycles of INO, 17 of 31 patients (55\%) achieved CR, nine (29\%) achieved PR, one patient (3\%) experienced early death and four patients with stable, resistant or progressive disease did not receive further INO treatment (13\%). Interestingly, only two patients with PR after the first cycle achieved a CR after the second INO cycle, whereas the other seven patients

Table 2. Localization of extramedullary disease.

\begin{tabular}{|l|c|}
\hline Localization of extramedullary disease* & Number \\
\hline Lymph nodes & 15 \\
\hline Gastrointestinal organs & 15 \\
\hline Osteolytic lesions & 12 \\
\hline Skin lesions & 7 \\
\hline Soft tissue & 5 \\
\hline Genitals & 4 \\
\hline Mediastinal mass & 2 \\
\hline Lung/pleural effusion & 2 \\
\hline Epidural mass & 2 \\
\hline Nasopharyngeal mass & 2 \\
\hline Central nervous system with epidural mass & 1 \\
\hline Peripheral nerves & 1 \\
\hline Vertebral mass & 1 \\
\hline Pelvic mass & 1 \\
\hline Cardiac involvement & 1 \\
\hline
\end{tabular}

* Overall, patients had in median two extramedullary disease manifestations (range, 1-9). Each localization of extramedullary disease was counted separately; thus, the total number does not add up to the total number of patients. 
A

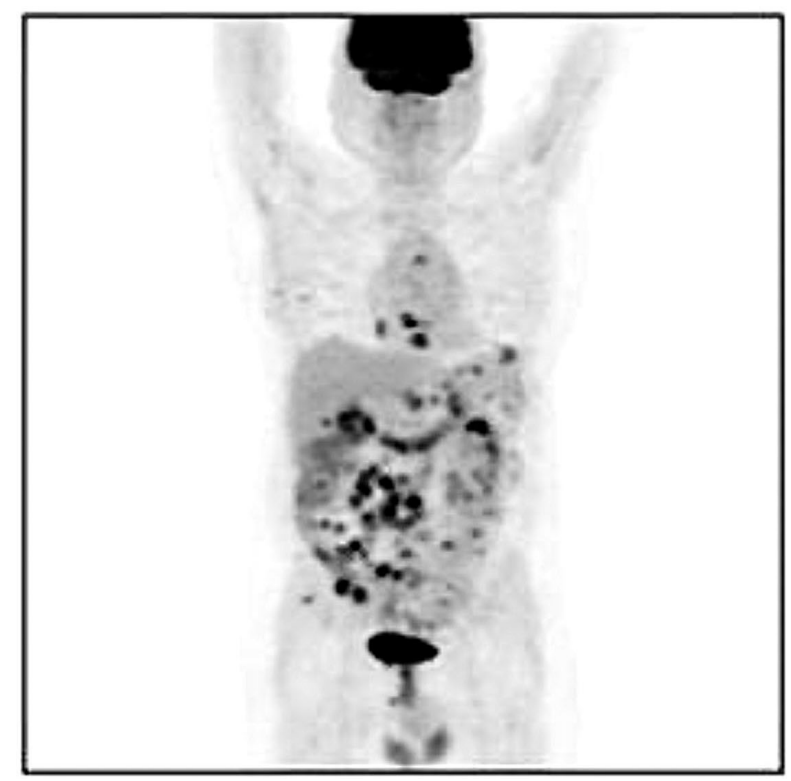

B

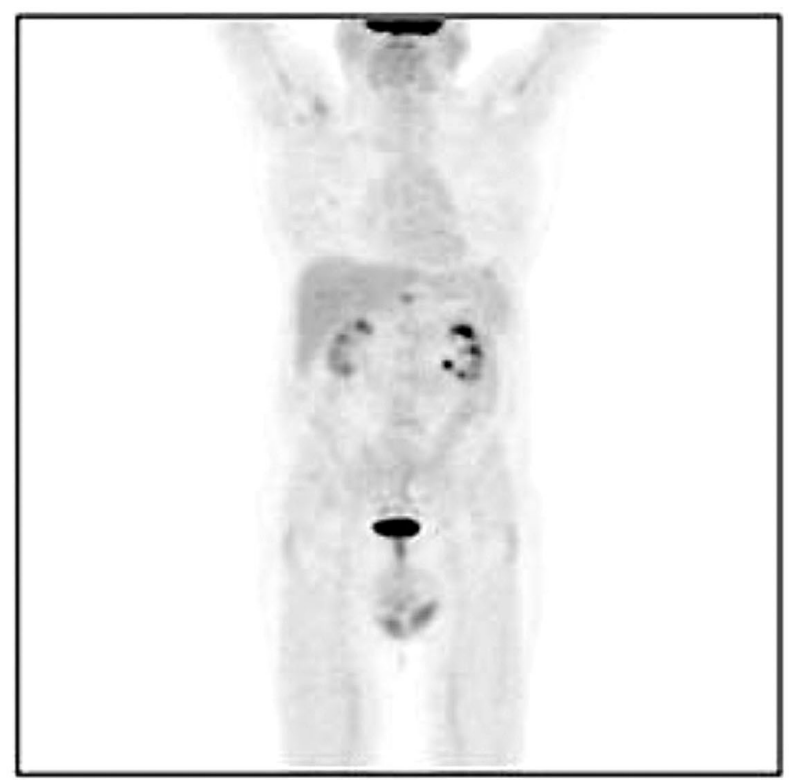

Figure 1. Whole body 18-fluorodeoxyglucose positron emission tomography-computed tomography. (A) Before the start of treatment with inotuzumab ozogamicin (B) After one cycle of inotuzumab ozogamicin, showing partial remission.

A

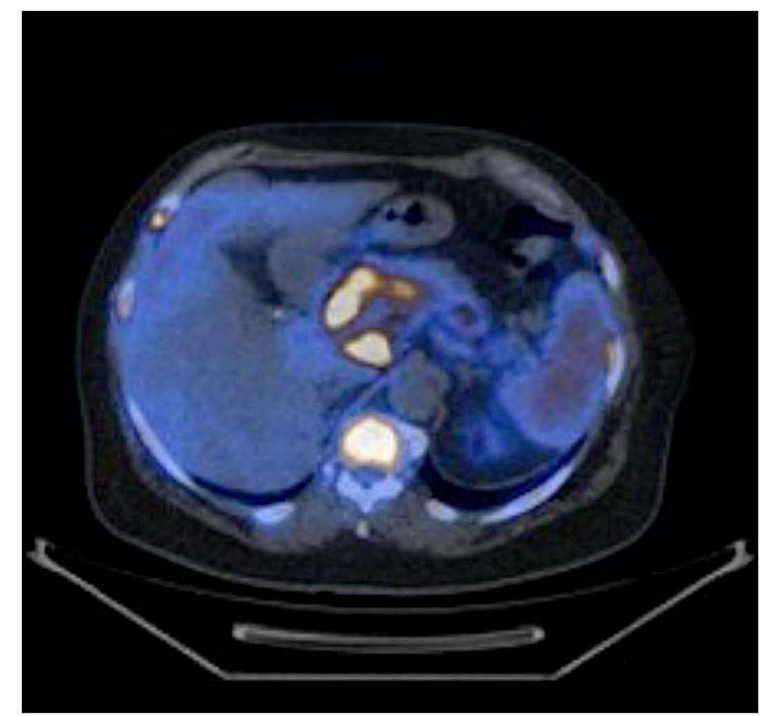

B

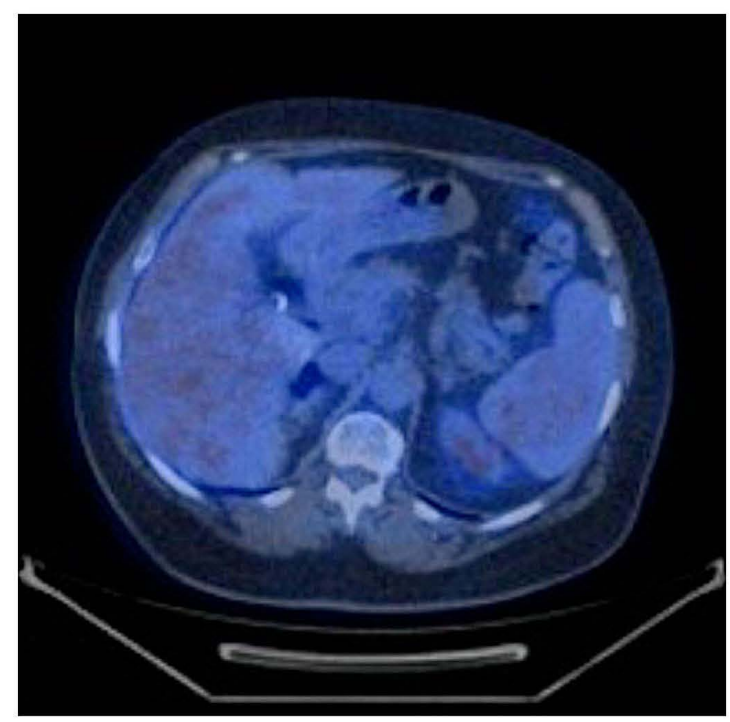

Figure 2. Contrast-enhanced imaging by positron emission tomography-computed tomography (axial slice). (A) Before the start of treatment with inotuzumab ozogamicin. (B) After one cycle of inotuzumab ozogamicin, showing complete remission.

with PR after the first cycle maintained the PR.

Patients, who achieved at least a PR after two INO cycles and did not proceed to allogeneic HSCT, could continue with INO for up to six cycles.

\section{Survival}

The median follow-up was 29 months (95\% Cl: 21 months - not reached) and the median OS was 12.8 months (95\% Cl: 9.9-16.2 months) (Figure 3). One-year and 2-years OS and relapse-free survival rates were 53\% (95\% Cl: $37-76 \%)$ and $47 \%(95 \% \mathrm{Cl}: 25-88 \%)$ and $18 \%(95 \% \mathrm{Cl}: 8-43 \%)$ and 23\% (95\% Cl: 7-75\%), respectively (Figure 4). In Cox regression analysis age as a continuous variable had no impact on OS ( $P=0.83)$. This was also true when using 60 years as cut-off $(P=0.2)$. Twelve patients went on to allogeneic HSCT ( $C R, n=6$; PR, $n=3$; progressive disease, $n=3$ ). Prior to allogeneic HSCT, eight patients received two or fewer cycles of INO and four patients received three or four INO cycles. The influence of allogeneic HSCT assessed as a time-dependent co-variable as post-remission therapy on OS is illustrated by a Simon Makuch plot (Figure 5). The Mantel-Byar test revealed no impact on OS $(P=0.19)$ for patients proceeding to allogeneic HSCT as compared to consolidation with INO. A multivariable Andersen-Gill model including prior allogeneic HSCT before INO treatment, age at initial diagnosis and allogeneic HSCT after INO as a time-dependent variable did not show any significant impact of any of these variables on OS.

In patients achieving a CR after INO treatment $(n=17)$, the median OS was 16.2 months. There was no difference in OS $(P=0.08)$ or relapse-free survival $(P=0.2)$ according to whether patients had EMD manifestations only as compared to EMD and bone marrow involvement.

Of the 26 patients in CR/PR after INO treatment, ten relapsed (38\%; after allogeneic HSCT, $n=3$ ); of those, all except one succumbed to their disease. Two patients died in remission (sepsis, VOD/SOS/multi-organ failure, $n=1$; each); both had undergone allogeneic HSCT before INO 


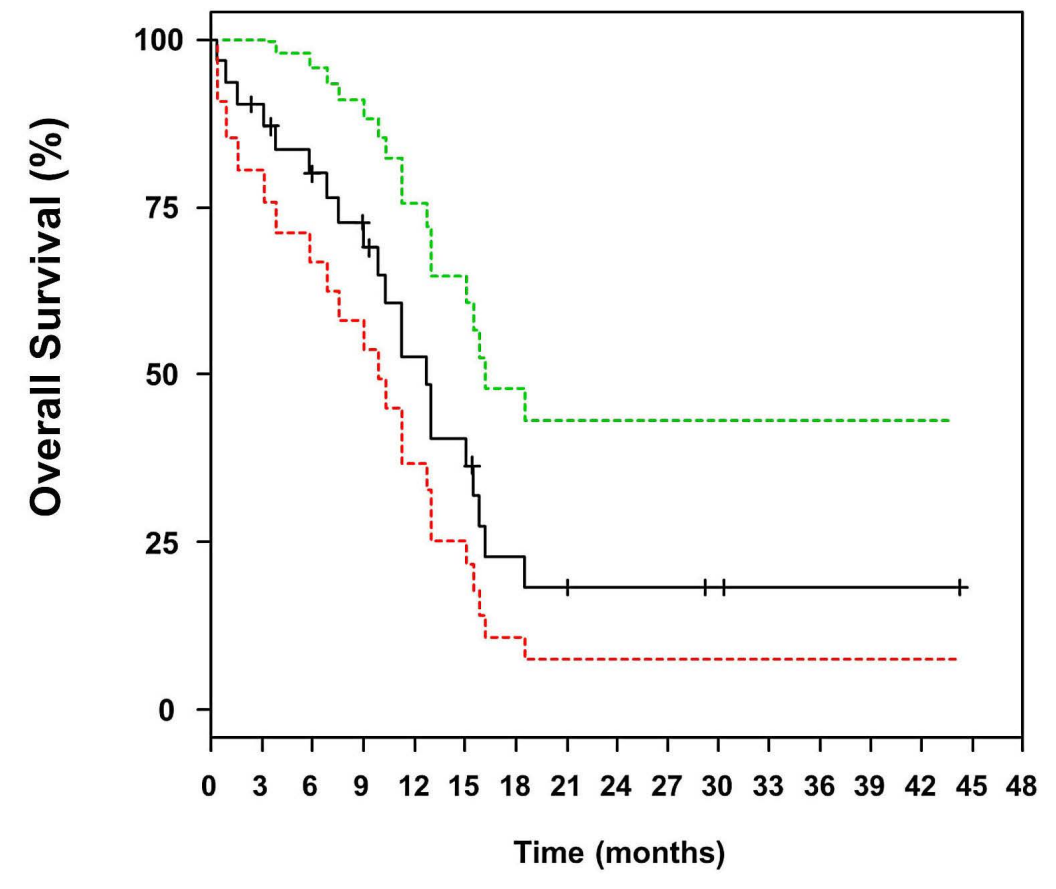

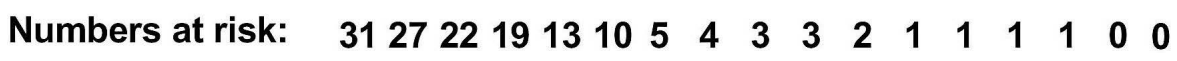

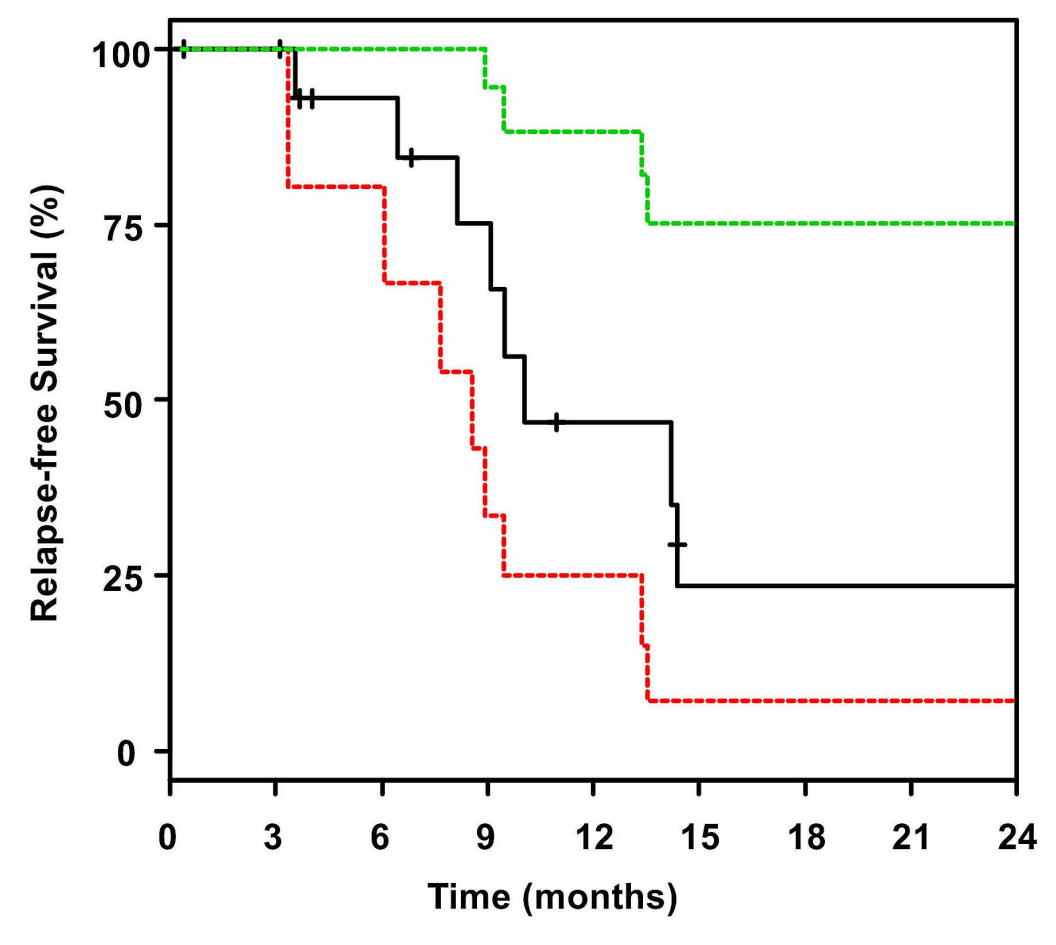

$\begin{array}{llllllllll}\text { Numbers at risk: } & 31 & 27 & 22 & 19 & 13 & 10 & 5 & 4 & 3\end{array}$
Figure 3. Overall survival of relapsed/refractory patients with B-acute lymphoblastic leukemia and extramedullary disease after treatment with Inotuzumab ozogamicin. Green and red dotted lines indicate upper and lower bounds of the $95 \%$ confidence interval.

Figure 4. Relapse-free survival of patients attaining complete remission. Green and red dotted lines indicate upper and lower bounds of the $95 \%$ confidence interval. treatment. One patient experienced a molecular relapse, which was successfully treated with INO again. Ten patients are still in CR $(n=9)$ or PR $(n=1)$, including the patient with prior molecular relapse and re-exposure to INO.

Our cohort also included three patients with central nervous system involvement. The first patient initially developed central nervous system relapse with positive cytology, but eventually progressed with an epidural mass treated with INO and ponatinib. This patient developed VOD after three INO cycles. Thus, all treatment was withheld. The cerebrospinal fluid remained intermittently positive for ALL (treated with intrathecal chemotherapy), but the peripheral blood remained negative and the epidural mass has not recurred. The second patient was treated with six cycles of intrathecal methotrexate/cytarabine/dexamethasone. The cerebrospinal fluid was negative after the second cycle and remained negative thereafter. The patient also received four INO cycles and achieved CR without measurable residual disease after the second INO cycle. The patient went on to allogeneic HSCT, but relapsed 3.5 months later and died 8.2 months after relapse. Finally, in the last patient, central nervous system relapse was not confirmed (both cerebrospinal fluid evaluation and magnetic resonance imaging were equivocal), but suspected due to diplopia, which improved after high-dose methotrexate (given before INO). The patient was then switched to six INO cycles and achieved CR according to PET-CT after three INO cycles. Unfortunately, the patient developed systemic (blood/marrow/extramedullary) relapse 1.5 months later. Cerebrospinal fluid at that time was negative and there were additionally no suggestive central nervous systems symptoms (no recurrence of diplopia or other neurological deficits). The patient died 13 days after relapse due to rapidly progressive disease. 


\section{Veno-occlusive disease and sinusoidal obstruction syndrome}

Up to four INO cycles were administered in patients as a bridge to transplantation ( $\leq 2$ cycles, $n=9 ; 3-4$ cycles, $n=3$ ). Overall, VOD/SOS occurred in three (10\%) patients, including one ( $8 \%$ ) of 12 patients after transplantation. The first patient experienced VOD on the first day of the third INO cycle prior to allogeneic HSCT, but continued to transplantation after resolution and is in ongoing CR 24 months after transplant. The second patient developed VOD after three INO cycles and therefore stopped INO treatment. This patient did not proceed to allogeneic HSCT and is in CR 30.7 months after the start of INO treatment. The third patient received two cycles of INO prior to haplo-identical allogeneic HSCT with a conditioning regimen consisting of treosulfan/fludarabine/thiotepa. This patient developed VOD after transplantation and died 13.1 months after the transplant due to multi-organ failure and VOD.

\section{Discussion}

EMD is reported to occur in $20 \%$ of patients with ALL, being more common in patients with a T-cell phenotype, as well as in patients presenting with lymphoblastic lymphoma, without bone marrow involvement. ${ }^{27}$ EMD may involve different sites, as observed in our series. ${ }^{28,29}$ The role of INO as treatment for patients with $r / r$ ALL and EMD has largely not been studied. The randomized phase III INOVATE trial included only seven $r / r$ ALL patients with EMD given INO treatment as well as five patients treated with standard-of-care chemotherapy. ${ }^{30}$ Among patients with baseline EMD, five of seven (71\%) in the INO arm and two of five (40\%) in the standard care arm achieved CR/CRi,

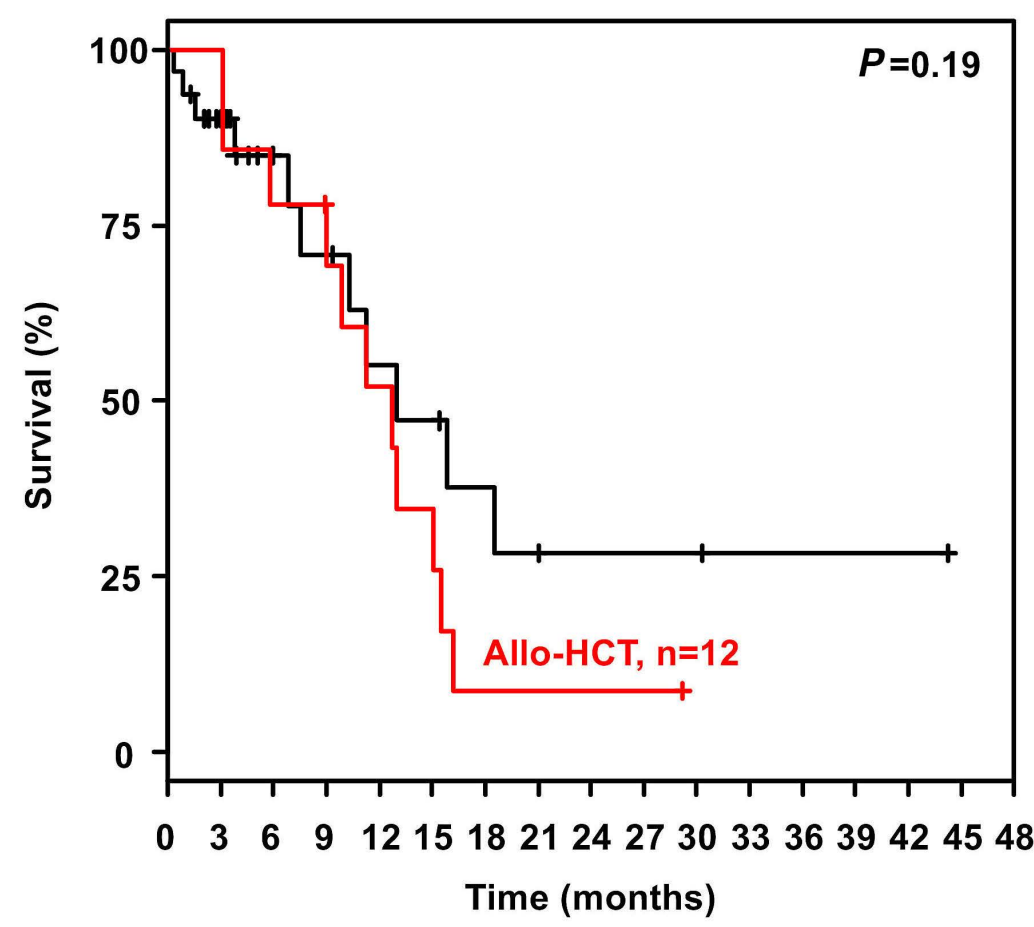

Numbers at risk: $\begin{array}{llllllllllllllllll}31 & 27 & 22 & 19 & 13 & 10 & 5 & 4 & 3 & 3 & 2 & 1 & 1 & 1 & 1 & 0 & 0\end{array}$ $\begin{array}{lllllllllllllllll}12 & 12 & 10 & 9 & 6 & 4 & 1 & 1 & 1 & 1 & 0 & 0 & 0 & 0 & 0 & 0 & 0\end{array}$ which included resolution of EMD. ${ }^{30}$ Consistent with previous reports on the effectiveness of INO in patients with EMD, we observed a high CR rate of $55 \%$ after INO treatment in patients with $r / r$ ALL and EMD. ${ }^{30-32}$ There was no difference based on the presence or absence of concurrent bone marrow disease. Additionally, the median os of 12.8 months in our cohort of heavily pretreated patients compares favorably to that of the less heavily pre-treated patients enrolled in the INO-VATE trial (7.7 months), although the number of patients with EMD in the aforementioned trial was very low limiting the comparison. ${ }^{31}$ The high response rate of ALL with EMD treated with INO may be an advantage of INO treatment since the presence or history of EMD may predict poor responses to other therapies, specifically blinatumomab..$^{19}$ In a retrospective cohort study of 65 patients with $\mathrm{r} / \mathrm{r}$ ALL, a high leukemia burden, defined as bone marrow blast cells $>50 \%$ (odds ratio $=0.24 ; P=0.02$ ) as well as presence of EMD (odds ratio $=0.19 ; P=0.05$ ) or history of EMD (odds ratio $=0.23$; $P=0.005)$ were associated with lower response to blinatumomab.19 It remains unknown whether increasing the dose of blinatumomab for ALL would be able to overcome this resistance (and be tolerable), since a higher dose has been studied for non-Hodgkin lymphomas and produced reasonable results. ${ }^{33}$ In contrast to blinatumomab, ${ }^{34}$ only a few cases of CD22 antigen loss have been described so far. ${ }^{35,36}$ In our cohort, we did not observe any CD22 antigen loss. In the INO-VATE trial, the inclusion of a small group of patients who were CD22-negative or had low CD22 expression was reported. Interestingly, three of five of these patients showed a response to INO treatment. ${ }^{15}$ Furthermore, response in a CD22-negative patient was also described in a case report. ${ }^{37}$ Thus, INO might be active in CD22-negative patients and/or those with very dim CD22

Figure 5. Simon Makuch plot illustrating the influence of allogeneic hematopoietic stem cell transplantation on overall survival. Allo-HCT: allogeneic hematopoietic stem cell transplantation. 
expression, but this remains to be elucidated in larger studies. Recent data from the INO-VATE trial suggest that patients with high ( $\geq 90 \%)$ CD22 expression levels had a higher CR rate compared to those with $<90 \%$ expression (42.1\% [n=45/107] vs. 20\% [n=7/35]). ${ }^{38}$ According to the European Medicine Agency label, CD22 expression needs to be above $0 \%$, thus also including patients with very dim CD22 expression levels. In the USA, the Food \& Drug Administration did not specific any particular CD22 expression.

Lineage switch (myeloid conversion), described mostly in patients receiving chimeric antigen receptor $\mathrm{T}$-cell therapy, does not seem to involve CD22 expression: the antigen is maintained in intermediate phenotype relapses, suggesting that simultaneous pressure on CD19 and CD22 might avoid this mechanism of resistance..$^{39}$

Increased exposure to INO has been associated with an increased risk of VOD/SOS following allogeneic HSCT, leading to the recommendation that patients being treated with INO as a bridge to allogeneic HSCT should be treated with two or fewer cycles of the drug ( 3 cycles if necessary to achieve a measurable residual diseasenegative CR/CRi). ${ }^{40-42}$ In our cohort, VOD/SOS occurred in only three patients, including one after allogeneic HSCT, although up to four INO cycles were administered prior to transplantation. These data compare favorably to previously reported data. ${ }^{15}$

Our analysis has several limitations. Since this is a retrospective, non-randomized cohort analysis no direct comparison to outcome of $r / r$ ALL with EMD after standard-of-care chemotherapy treatment was feasible. However, since all patients were heavily pretreated with intensive chemotherapy including prior allogeneic HSCT in $58 \%$ of the patients, we believe that standard-of-care chemotherapy would have failed to induce a remission. The overall prognosis remains poor even if patients could be successfully bridged to allogeneic HSCT, strongly arguing for alternative consolidation approaches, such as chimeric antigen receptor $\mathrm{T}$ cells or advanced bi-specific antibodies. ${ }^{43}$ Nevertheless, the ability of INO to be given in an outpatient setting with few toxicities may continue to make it a valuable possibility in the treatment of B-ALL. In conclusion, this outcome analysis demonstrates that treatment with INO is an effective and promising approach in $r / r$ ALL patients with EMD. The CD22 status should be routinely assessed at diagnosis and $r / r$ B-ALL patients, in order to evaluate the indication for INO treatment better. However, allogenic HSCT alone seems not to be effective in maintaining disease control. Thus, chimeric antigen receptor $\mathrm{T}$ cells or advanced bi-specific antibodies as consolidation therapy should be evaluated in the future.

\section{Disclosures}

No conflicts of interest to disclose.

\section{Contributions}

SK and RFS were responsible for the concept of this study, contributed to the literature search, collection, analysis and interpretation of the data, and wrote the manuscript. $C P$ was responsible for the concept of the study, contributed to the literature search and data collection, contributed patients, analyzed and interpreted data, and critically revised the manuscript. NP analyzed and interpreted data. CS, MRL JW, FG, AMB, MF, CL, DW, ADH and $M J L$ contributed patients and critically revised the manuscript. All authors reviewed and approved the final version of the manuscript.

\section{Funding}

SK was supported by the Olympia-Morata fellowship program from the Medical Faculty of Heidelberg University. We acknowledge publication support from Leipzig University.

\section{Data-sharing statement}

Questions regarding data sharing should be addressed to the corresponding author.

\section{References}

1. Hoelzer D, Bassan R, Dombret $\mathrm{H}$, et al. Acute lymphoblastic leukaemia in adult patients: ESMO clinical practice guidelines for diagnosis, treatment and follow-up. Ann Oncol.

2016;27(Suppl 5):v69-82.

2. Spinelli O, Peruta B, Tosi M, et al. Clearance of minimal residual disease after allogeneic stem cell transplantation and the prediction of the clinical outcome of adult patients with highrisk acute lymphoblastic leukemia. Haematologica. 2007;92(5):612-618.

3. Schrappe M. Detection and management of minimal residual disease in acute lymphoblastic leukemia. Hematology Am Soc Hematol Educ Program. 2014;2014(1):244-249.

4. Davila ML, Riviere I, Wang X, et al. Efficacy and toxicity management of 19-28z CAR T cell therapy in B cell acute lymphoblastic leukemia. Sci Transl Med. 2014;6(224):224ra225.

5. Cruz CR, Micklethwaite KP, Savoldo B, et al. Infusion of donorderived CD19-redirected virus-specific $T$ cells for B-cell malignancies relapsed after allogeneic stem cell transplant: a phase 1 study. Blood. 2013;122(17):2965-2973.

6. Jacoby E. The role of allogeneic HSCT after CAR T cells for acute lymphoblastic leukemia. Bone Marrow Transplant. 2019;54(Suppl 2):810-814.

7. Kantarjian HM, Thomas D, Ravandi F, et al. Defining the course and prognosis of adults with acute lymphocytic leukemia in first salvage after induction failure or short first remission duration. Cancer. 2010;116(24):5568-5574. 
8. Thomas DA, Kantarjian H, Smith TL, et al. Primary refractory and relapsed adult acute lymphoblastic leukemia: characteristics, treatment results, and prognosis with salvage therapy. Cancer. 1999;86(7):1216-1230.

9. Oriol A, Vives S, Hernández-Rivas JM, et al. Outcome after relapse of acute lymphoblastic leukemia in adult patients included in four consecutive risk-adapted trials by the PETHEMA Study Group. Haematologica. 2010;95(4):589-596.

10. Tavernier E, Boiron JM, Huguet F, et al. Outcome of treatment after first relapse in adults with acute lymphoblastic leukemia initially treated by the LALA-94 trial. Leukemia. 2007;21(9):1907-1914.

11. Gökbuget N, Stanze D, Beck J, et al. Outcome of relapsed adult lymphoblastic leukemia depends on response to salvage chemotherapy, prognostic factors, and performance of stem cell transplantation. Blood. 2012;120(10):2032-2041.

12. Specchia G, Pastore D, Carluccio P, et al. FLAG-IDA in the treatment of refractory/relapsed adult acute lymphoblastic leukemia. Ann Hematol. 2005;84(12):792-795.

13. O'Brien S, Schiller G, Lister J, et al. High-dose vincristine sulfate liposome injection for advanced, relapsed, and refractory adult Philadelphia chromosome negative acute lymphoblastic leukemia. J Clin Oncol. 2013;31(6):676-683.

14. Kantarjian $H$, Stein A, Gokbuget N, et al. Blinatumomab versus chemotherapy for advanced acute lymphoblastic leukemia. $\mathrm{N}$ Engl J Med. 2017;376(9):836-847.

15. Kantarjian HM, DeAngelo DJ, Stelljes M, et al. Inotuzumab ozogamicin versus standard therapy for acute lymphoblastic leukemia. N Engl J Med. 2016;375(9):740-753.

16. DiJoseph JF, Armellino DC, Boghaert ER, et al. Antibody-targeted chemotherapy with CMC-544: a CD22-targeted immunoconjugate of calicheamicin for the treatment of Blymphoid malignancies. Blood. 2004;103(5):1807-1814.

17. Shor B, Gerber HP, Sapra P. Preclinical and clinical development of Inotuzumab-ozogamicin in hematological malignancies. Mol Immunol. 2015;67(2 Pt A):107-116.

18. Lau KM, Saunders IM, Goodman AM. Characterization of relapse patterns in patients with acute lymphoblastic leukemia treated with blinatumomab. J Oncol Pharm Pract. 2021;27(4):821-826.

19. Aldoss I, Song J, Stiller T, et al. Correlates of resistance and relapse during blinatumomab therapy for relapsed/refractory acute lymphoblastic leukemia. Am J Hematol. 2017;92(9):858-865.

20. Mitelman F. ISCN: An International System for Human Cytogenetic Nomenclature. S. Karger: Basel, Switzerland;1995.

21. Schemper M, Smith TL. A note on quantifying follow-up in studies of failure time. Control Clin Trials. 1996;17(4):343-346.

22. Kaplan E, Meier P. Nonparametric estimation from incomplete observations. J Am Stat Assoc. 1958;53(282):457-481.

23. Mantel N, Byar D. Evaluation of response-time data involving transient states: an illustration using heart transplant data. $J$ Am Stat Assoc. 1974;69(345):81-86.

24. Simon R, Makuch RW. A non-parametric graphical representation of the relationship between survival and the occurrence of an event: application to responder versus non-responder bias. Stat Med. 1984;3(1):35-44.

25. Andersen P, Gill RD. Cox's regression model for counting processes: a large sample study. Ann Stat. 1982;10(4):1100-1120.

26. $R$ Development Core Team. R: A language and environment for statistical computing. R Foundation for Statistical Computing: Vienna, Austria, 2014.

27. Geethakumari PR, Hoffmann MS, Pemmaraju N, et al. Extramedullary B lymphoblastic leukemia/lymphoma (B-ALL/BLBL): a diagnostic challenge. Clin Lymphoma Myeloma Leuk. 2014;14(4):e115-e118.

28. Silva WFD, Marquez GL, Salim RC, Rocha V. Primary refractory Bcell lymphoblastic leukemia with extramedullary disease - a distinctive response to blinatumomab and inotuzumab ozogamicin. Hematol Transfus Cell Ther. 2019;41(4):356-359.

29. Lucas DR, Bentley G, Dan ME, Tabaczka P, Poulik JM, Mott MP. Ewing sarcoma vs lymphoblastic lymphoma. Am J Clin Pathol. 2001;115(1):11-17.

30. DeAngelo DJ, Advani AS, Marks DI, et al. Inotuzumab ozogamicin for relapsed/refractory acute lymphoblastic leukemia: outcomes by disease burden. Blood Cancer J. 2020;10(8):81.

31. Bertamini L, Nanni J, Marconi G, et al. Inotuzumab ozogamicin is effective in relapsed/refractory extramedullary B acute lymphoblastic leukemia. BMC Cancer. 2018;18(1):1117.

32. Lee SH, Yoon JH, Min GJ, et al. Response to blinatumomab or inotuzumab ozogamicin for isolated extramedullary relapse of adult acute lymphoblastic leukemia after allogeneic hematopoietic cell transplantation: a case study. Int J Hematol. 2022;115(1):135-139.

33. Sanders S, Stewart DA. Targeting non-Hodgkin lymphoma with blinatumomab. Expert Opin Biol Ther. 2017;17(8):1013-1017.

34. Ruella M, Maus MV. Catch me if you can: leukemia escape after CD19-directed T cell immunotherapies. Comput Struct Biotechnol J. 2016;14:357-362.

35. Jacoby E, Nguyen SM, Fountaine TJ, et al. CD19 CAR immune pressure induces B-precursor acute lymphoblastic leukaemia lineage switch exposing inherent leukaemic plasticity. Nat Commun. 2016;7:12320.

36. Paul MR, Wong V, Aristizabal P, Kuo DJ. Treatment of recurrent refractory pediatric pre-B acute lymphoblastic leukemia using inotuzumab ozogamicin monotherapy resulting in CD22 antigen expression loss as a mechanism of therapy resistance. $\mathrm{J}$ Pediatr Hematol Oncol. 2019;41(8):e546-e549.

37. Reinert J, Beitzen-Heineke A, Wethmar K, Stelljes M, Fiedler W, Schwartz S. Loss of CD22 expression and expansion of a CD22 dim subpopulation in adults with relapsed/refractory Blymphoblastic leukaemia after treatment with Inotuzumab-Ozogamicin. Ann Hematol. 2021;100(11):2727-2732.

38. Kantarjian HM, Stock W, Cassaday RD, et al. Inotuzumab ozogamicin for relapsed/refractory acute lymphoblastic leukemia in the INO-VATE trial: CD22 pharmacodynamics, efficacy, and safety by baseline CD22. Clin Cancer Res. 2021;27(10):2742-2754.

39. Fingrut W, Davis W, Dallas K, et al. Inotuzumab ozogamicin as salvage therapy for relapsed $\mathrm{B}$-cell acute lymphoblastic leukemia with only very dim expression of $\mathrm{Cd} 22$ on circulating blasts. A case report. Clin Lymphoma Myeloma Leuk. 2019;19:S184.

40. Kantarjian HM, DeAngelo DJ, Advani AS, et al. Hepatic adverse event profile of Inotuzumab ozogamicin in adult patients with relapsed or refractory acute lymphoblastic leukaemia: results from the open-label, randomised, phase 3 INO-VATE study. Lancet Haematol. 2017;4(8):e387-e398.

41. Marks DI, Kebriaei P, Stelljes M, et al. Outcomes of allogeneic stem cell transplantation after inotuzumab ozogamicin treatment for relapsed or refractory acute lymphoblastic leukemia. Biol Blood Marrow Transplant. 2019;25(9):1720-1729.

42. Kebriaei P, Cutler C, de Lima M, et al. Management of important adverse events associated with inotuzumab ozogamicin: expert panel review. Bone Marrow Transplant. 2018;53(4):449-456.

43. Wan X, Yang X, Yang F, et al. Outcomes of anti-CD19 CAR-T treatment of pediatric B-ALL with bone marrow and extramedullary relapse. Cancer Res Treat. 2022;54(3):917-925. 